\title{
A Novel CubeSat-Sized Antiproton Detector for Space Applications
}

\section{Thomas Pöschl*}

Technische Universität München

Physik-Department E18, James-Franck-Str 1, 85748 Garching, Germany

E-mail: thomas.poeschleph.tum.de

\section{Martin J. Losekamm}

Technische Universität München

Physik-Department E18, James-Franck-Str 1, 85748 Garching, Germany

Institute of Astronautics, Boltzmannstr. 15, 85748 Garching, Germany

E-mail: m. losekammatum.de

\section{Daniel Greenwald}

Technische Universität München

Physik-Department E18, James-Franck-Str 1, 85748 Garching, Germany

\section{Stephan Paul}

Technische Universität München

Physik-Department E18, James-Franck-Str 1, 85748 Garching, Germany

\begin{abstract}
Cosmic antimatter is an excellent probe for many astrophysical processes. The abundancies and energy spectra of antiparticles help to understand the creation and propagation mechanisms of cosmic ray particles in the universe. The flux of geomagnetically trapped antiprotons in Earth's inner radiation belt can help to verify our models of the production of secondary cosmic ray particles and their motion in Earth's magnetic field. The Antiproton Flux in Space (AFIS) experiment will measure this flux using a novel CubeSat-sized particle detector. This active-target detector consists of 900 scintillating fibers read out by silicon photomultipliers and is sensitive to antiprotons in the energy range below $100 \mathrm{MeV}$. It has a large geometrical acceptance of about $250 \mathrm{~cm}^{2} \mathrm{sr}$ because of its isotropic acceptance for particles. The particle identification scheme for antiprotons relies on a combination of Bragg curve spectroscopy and the characteristics of the annihilation process. In order to verify the detection principle, a prototype detector with a reduced number of channels was tested at a stationary proton beam. Its energy resolution was found to be less than $1 \mathrm{MeV}$ for stopping protons of about $50 \mathrm{MeV}$ energy. We give an overview of the AFIS mission and explain the working principle of the detector. We also discuss the results from the beam test.
\end{abstract}

The 34th International Cosmic Ray Conference,

30 July- 6 August, 2015

The Hague, The Netherlands

\footnotetext{
* Speaker.
} 


\section{Introduction}

Particles created in Earth's atmosphere can be trapped in belt-like structures around Earth by the terrestrial magnetic field. These so-called Van Allen belts—named after their discoverer James A. Van Allen - consist mainly of electrons and protons and were first detected in 1958 [18, 19]. Many have predicted the existence of a similar structure for antiprotons $[6,14,8]$. Due to the tilt and shift of Earth's magnetic dipole field from its rotational axis, the terrestrial magnetic field strength is asymmetrically dependent on altitude around the planet [13] with an area of depression over the southern Atlantic Ocean. In this so-called South Atlantic Anomaly (SAA), charged particles of the inner Van Allen belt penetrate the atmosphere down to altitudes of $200 \mathrm{~km}$. This is the only area in which a spacecraft in low-Earth orbit (LEO) can investigate this inner radiation belt.

The PAMELA experiment published the first measurements of the flux of trapped antiprotons in the SAA (Fig. 1) in 2011 [1]. Their measurements, based on 28 trapped antiprotons at energies above approximately $80 \mathrm{MeV}$, are two orders of magnitude smaller than theoretical predictions [14]. The AFIS experiment will complement this result by measuring the flux of trapped antiprotons in the range of $25 \mathrm{MeV}$ to $100 \mathrm{MeV}$ with a new active-target particle detector aboard a nanosatellite in the SAA.

In this paper we report the status of the experiment: section 2 describes the AFIS detector apparatus, and our expected signal for antiprotons; and section 3 describes the response of a scaled-down prototype to protons and an energy reconstruction scheme based on Bayesian inference techniques.

\section{The Multi-purpose Active-target Particle Telescope}

The centerpiece of the AFIS mission is the multi-purpose active-target particle telescope (MAPT).

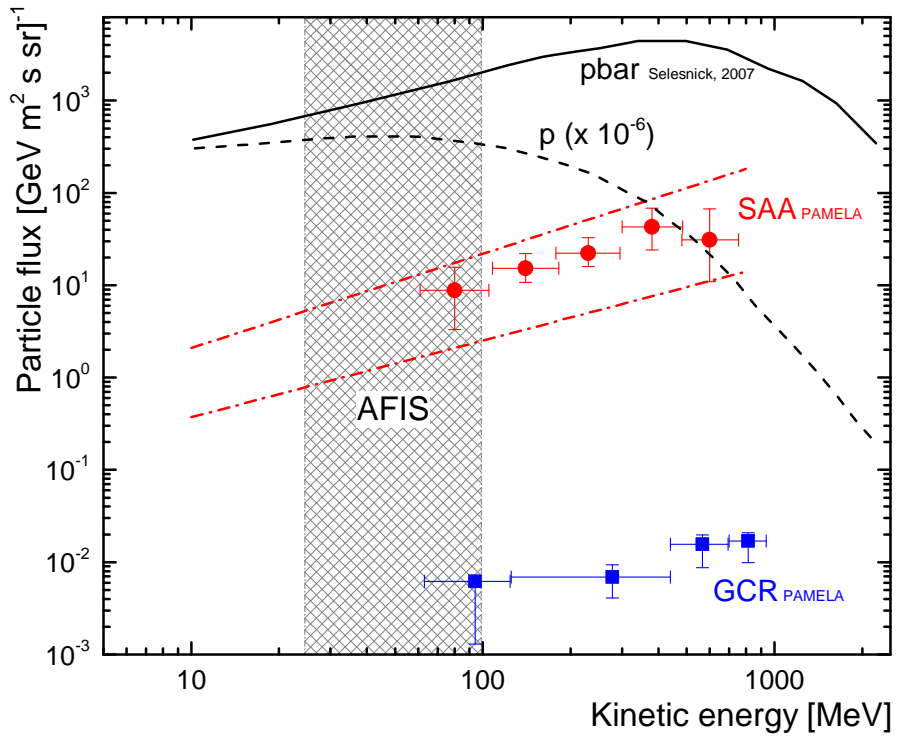

Figure 1: Fluxes of cosmic ray and trapped antiprotons inside the SAA as measured by the PAMELA experiment [1]; and theoretical predictions from [14]. The AFIS measurement region $(25 \mathrm{MeV}-100 \mathrm{MeV})$ is highlighted. 

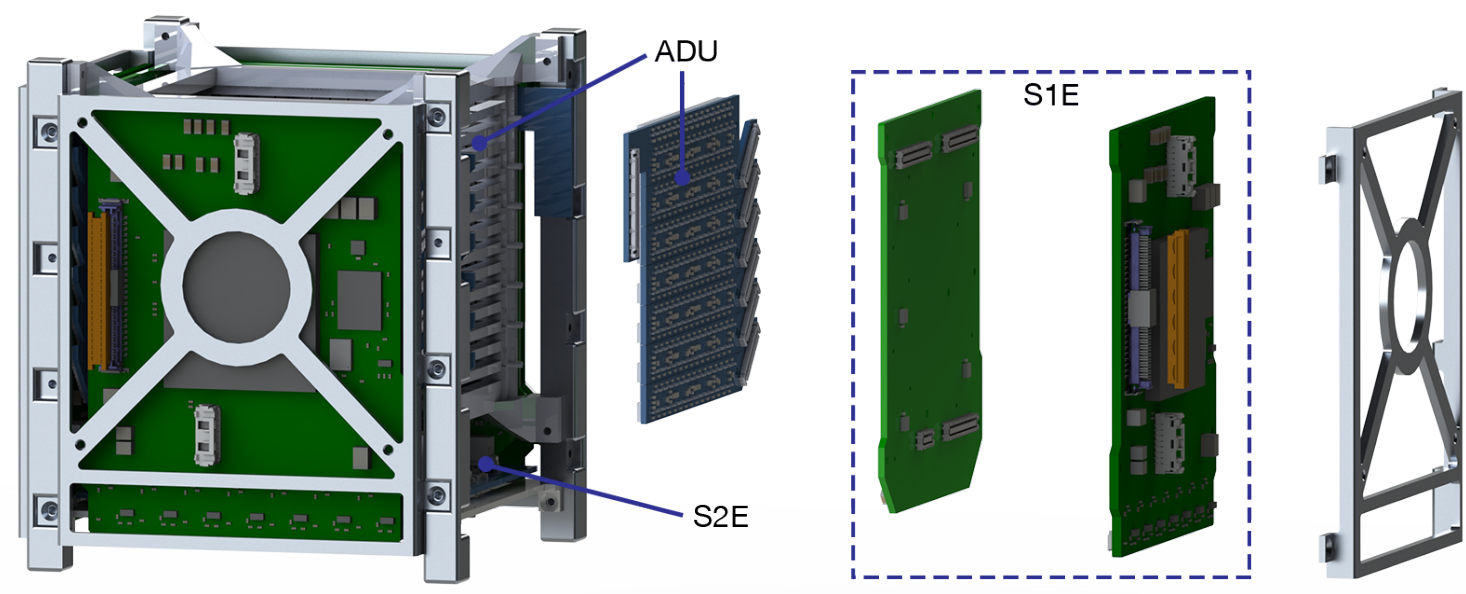

Figure 2: Structural layout of the MAPT. The ADU is symmetrically surrounded by the front-end electronics (S1E, S2E).

Its core, the so-called active-detection unit (ADU), is made of 900 scintillating plastic fibers arranged in 30 layers of 30 fibers each. Layers are rotated $90^{\circ}$ from their neighbors to allow 3D tracking of charged particles. This setup allows an isotropic acceptance for particles resulting in a large geometrical acceptance of about $250 \mathrm{~cm}^{2} \mathrm{sr}$ [11]. Each fiber is read out by a dedicated silicon photomultiplier (SiPM) with a matched peak-sensitivity wavelength. We use a 3D-printed frame to support the active core and mount it into the satellite structure that is built according to the CubeSat standard [17] (see Fig. 2).

Processing of the SiPM signals and their digitization is accomplished by the front-end electronics embedded in the detector module. To accept the very high event rates inside the SAA, signal processing of the individual channels is fully parallelized.

Twenty ASICs perform over-threshold analog-to-digital conversion of the signals [11]. Four FPGAs perform the subsequent time-over-threshold measurement. We can measure the signals of the SiPMs, which are $10 \mathrm{~ns}$ to $100 \mathrm{~ns}$ long, with a resolution of about $500 \mathrm{ps}$. A fifth FPGA combines the data and makes the trigger decision. The total power consumption of this setup is less than $20 \mathrm{~W}$, meeting the operational constraints aboard a nanosatellite.

\subsection{Particle Identification}

Unlike spectrometer-based detectors, the MAPT detector cannot directly determine a particle's rigidity or charge sign. Instead, we identify particle species by their stopping characteristics. We cannot use this technique to separate particle from antiparticle; but we can identify antiparticles by their characteristic annihilations: they create high-energy secondary particles originating from a single vertex. We can clearly identify an antiproton if

- it stops in the detector, so we can measure its Bragg curve and distinguish it from a highenergy particle;

- it annihilates inside the active detector volume; and

- its annihilation products include charged particles. 


\subsubsection{Bragg Curve Spectroscopy}

A particle with energy below the minimal ionizing region $(\beta \gamma \sim 3)$ initially deposits a small fraction of its energy per unit length in a medium. As its kinetic energy decreases, the stopping power of the medium on it increases and the particle deposits an increasing fraction of its energy. This increase proceeds more rapidly as the particle slows down, leading to the deposition of a significant fraction of the particle's energy at the end of its trajectory. The differential profile of such energy deposition as a function of depth is called the Bragg curve [10]. It is a distinct function of energy for each interacting particle species. Since the particle is completely stopped, the integral of the Bragg curve is the initial kinetic energy of the particle. The depth at which the particle stops, its range, depends on its charge number, atomic number, and initial velocity ${ }^{1}$. The maximum energy loss per unit distance depends only on the total charge of the incoming particle [7]. These dependencies can be exploited to identify the stopping particle. This technique is called Bragg curve spectroscopy and is widely used in nuclear and medical physics [7].

The MAPT detector cannot directly measure the energy loss per unit length. Instead, it measures the integral of the Bragg curve discretized by the fibers along the particle's track. Bragg curve spectroscopy therefore requires accurate track fitting to extract the true track length per channel and the total range of the particle inside the detector volume. Additionally, dead layers in the detector volume lead to differences between the deposited and measured energies and must be accounted for.

We can use this method to distinguish protons and antiprotons from other particles by their stopping and reconstruct their initial kinetic energies. However, such Bragg curve spectroscopy cannot distinguish between particle and antiparticle ${ }^{2}$. We will use characteristics of the annihilation process of the antiproton to identify it.

For particles that do not completely stop in the detector, we can use an extension of this technique for species identification based on the so-called $\Delta E-\Delta E$ method [16], which also cannot be used to discern particle from antiparticle. It can be applied to particles that pass completely through the detector without stopping, but also to antiprotons that annihilate in flight. The change of the energy deposition from channel to channel along the particle's track is used to extrapolate the stopping curve and subsequently determine the particle's characteristics. The extrapolation is based on fitting to the data values predicted from the Bethe equation, stopping-power tables, or a parameterization of the $\Delta E-\Delta E$ space [16]. A first study of the capabilities of these methods using test-beam data is discussed in section 3 .

\subsubsection{Antiproton Annihilation}

The most obvious difference between protons and antiprotons is that the latter annihilate with ordinary matter: the antiproton vanishes along with a nucleon of the surrounding matter, and the annihilated mass (about $2 \mathrm{GeV}$ ) is converted into energy released in the form of new secondary particles emerging from the reaction. If these particles are charged, we can see them as tracks inside the detector volume. Since the cross section for annihilation increases rapidly with decreasing particle velocity, the annihilation process usually takes place at very-low kinetic energies. Most

\footnotetext{
${ }^{1}$ The dependency on the atomic number and initial energy arises from the energy dependence.

${ }^{2}$ Neglecting the Barkas Effect occurring at very low particle energies [2], which we cannot observe.
} 
antiprotons will first slow down in the detector due to energy losses by ionization processes before annihilating into secondary particles emitted isotropically due to conservation of momentum. In contrast, background arising from high-energy proton interactions create secondary particles boosted into the initial direction of the proton. Approximately four detectable secondary particles are created per annihilation event. Most are fast pions, but heavier nuclear fragments and nucleons are also created via fragmentation of the matter nucleus ${ }^{3}$. While the pions are usually minimally ionizing and escape the detector without depositing a significant fraction of their energy, the heavier particles completely deposit their energies in the detector. This leads to a unique signature for the antiproton in its total energy deposition and its energy distribution in the detector volume. These characteristics are very rarely present in other high-energy reactions.

\section{In-Beam Measurement of Low-Energy Protons}

To evaluate the reconstruction of stopping particles and to investigate the performance of the fiber-SiPM combination, we tested a scaled-down prototype detector in a low-energy proton beam at the $\pi \mathrm{M} 1$ beam line of the Paul Scherrer Institute in Villigen, Switzerland, in late 2013. The prototype has 128 channels arranged in 16 layers of 8 fibers each. Like in the MAPT, layers are rotated $90^{\circ}$ from their neighbors. The central overlap region of the detector is $16 \mathrm{~mm}$ by $16 \mathrm{~mm}$ in cross section. We used scintillating plastic fibers of type SCSF-78 manufactured by Kuraray. To increase the light yield and prevent optical cross talk, we wrapped them in aluminum foil and mirrored them at one end. The SiPMs are glued to the fibers with optical glue on the unmirrored end. We use PM3350 silicon photomultipliers manufactured by KETEK with an active area of $3 \mathrm{~mm} \times 3 \mathrm{~mm}$ and a typical breakdown voltage of about $27 \mathrm{~V}$. Signals were digitized by eight mezzanine sampling analog-to-digital converters (MSADCs) developed for the COMPASS experiment at CERN [12] and triggered externally by an NaI scintillator in front of the detector.

\subsection{Event Reconstruction}

We calibrated the pulse height in each channel, and thus its gain, using a beam of minimumionizing particles (MIPs) - a $375-\mathrm{MeV} / \mathrm{c} \pi^{-}$beam. A MIP deposits approximately $0.4 \mathrm{MeV}$ in each layer of the detector and traverses all layers. We reconstruct the tracks of the particles in the detector using a track-finding algorithm based on a standard Hough transformation [9]. The response of the detector to protons was evaluated by recording the signal-depth distributions at four different beam momenta (see Fig. 3a).

Only about half of the kinetic energy of each stopping proton is measured in the detector. To quantify the loss mechanisms and to reconstruct the initial energies of incoming protons, we developed a numerical model based on the range tables provided by the NIST Physical Measurement Laboratory [3]. We took two important processes affecting the response of the detector into account: First, quenching of the organic scintillator at high ionization rates leads to a reduced light yield. According to Birk's empirical formula, the light yield depends on the ionization rate [4]:

$$
\left\langle\frac{\mathrm{d} L}{\mathrm{~d} x}\right\rangle \propto \frac{\left\langle\frac{\mathrm{d} E}{\mathrm{~d} x}\right\rangle}{1+k B\left\langle\frac{\mathrm{d} E}{\mathrm{~d} x}\right\rangle},
$$

\footnotetext{
${ }^{3}$ The antiproton can annihilate with both free protons-hydrogen nuclei-and larger nuclei.
} 


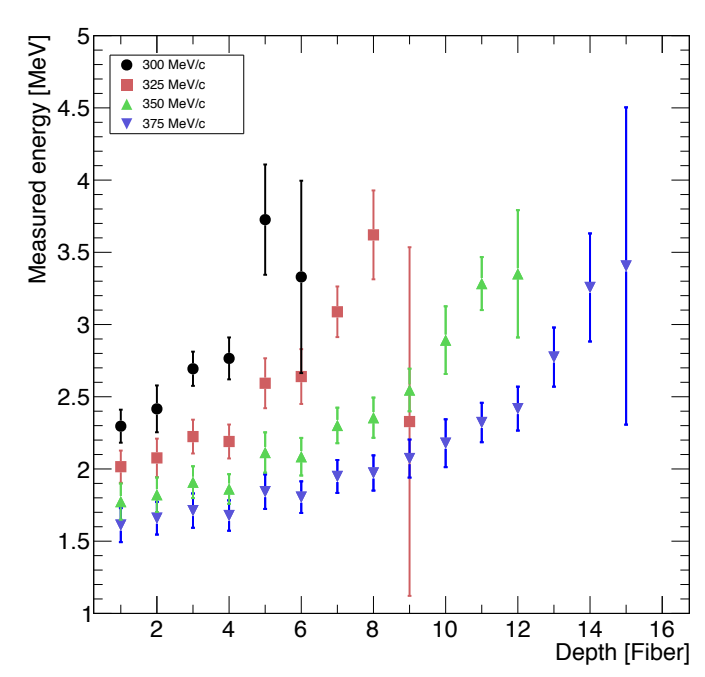

(a) Distribution of the measured energy deposition for different proton beam momenta.

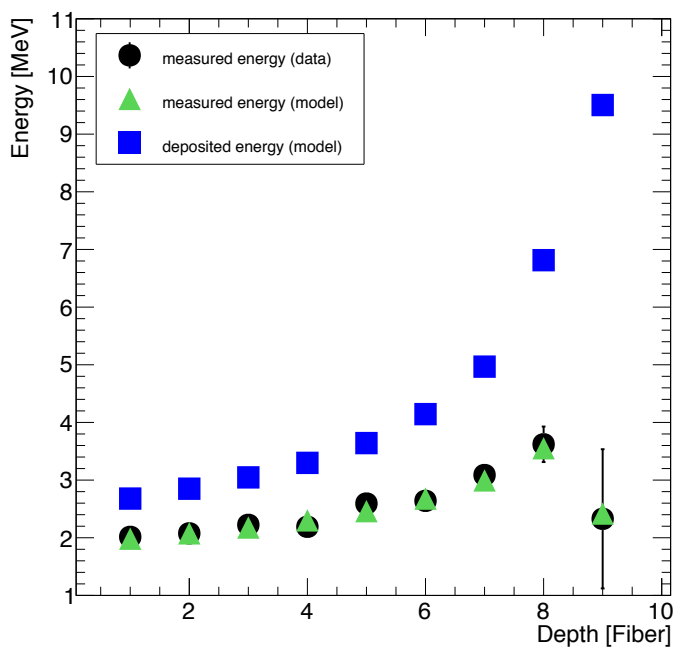

(b) Comparison of deposited and measured energies, for $325-\mathrm{MeV} / \mathrm{c}$ protons.

Figure 3: Response of the detector to stopping protons.

with $\langle\mathrm{d} L / \mathrm{d} x\rangle$ the light yield of the scintillator per unit length, $\langle\mathrm{d} E / \mathrm{d} x\rangle$ the energy loss of the traversing charged particle per unit length, and $k B$ Birks' coefficient. The latter is a material-specific constant and depends on the base material and the doping characteristics of the scintillator [15]. Since it has not yet been measured experimentally for our material, we treated it as a free parameter in our analysis.

The second loss mechanism is the saturation of the SiPMs at high light exposure, experienced during low-energy proton events. It introduces an additional nonlinear behavior in the measurement and was accounted for in the analysis as well.

\subsection{Bayesian Analysis}

We use the Bayesian Analysis Toolkit (BAT) software package [5] to compare the measured data with the expectations from our numerical model using Bayesian inference techniques. The posterior likelihood distributions of unknown parameters and their correlations can be evaluated using Markov Chain Monte Carlo (MCMC) methods [5]. To calculate the probability distributions for the free parameters, such as the initial energies of the particles and Birks' coefficient, their values are varied and the logarithmic likelihood (log-likelihood) function for each set of parameters is evaluated. We define the overall log-likelihood function as the sum of the log-likelihood functions for all fiber layers:

$$
\ln L=\sum_{i} \ln L^{(i)}\left(S_{\text {meas }}^{(i)} \mid S_{\text {pred }}^{(i)}\right)
$$

with $S_{\text {pred }}^{(i)}$ being the predicted signal and $S_{\text {meas }}^{(i)}$ the measured signal in the $i$ th layer. The loglikelihood function for a single fiber is calculated by evaluating the logarithm of the probability of the data set given the prediction, which we calculate as the likelihood of the prediction given the spread of the data. The measured distribution can be approximated by a Gaussian distribution with median $\mu$ and standard deviation $\sigma$. Both values are obtained by fitting the measured pulse height 
spectra in each layer. Hence, we calculate

$$
\begin{aligned}
& \ln L^{(i)}\left(S_{\text {meas }}^{(i)} \mid S_{\text {pred }}^{(i)}\right)=-\frac{1}{2}\left(\frac{x^{(i)}-\mu^{(i)}}{\sigma^{(i)}}\right)^{2} \\
& -\ln \left(\sqrt{2 \pi} \sigma^{(i)}\right) \underbrace{+\ln 2}_{\text {for non-hit layers }}
\end{aligned}
$$

with $x^{(i)}$ being the predicted median signal height in the $i$ th fiber. Per definition, the signal value is always positive. This formula is only valid if the measured distribution is far away from the origin. In our case, this requirement is always fulfilled except for layers that were not hit. In the calculation of the log-likelihood function for these layers, an additional factor of $\ln 2$ must be added.

\subsection{Results}

Using the MCMC method, we obtained the posterior probability distributions of the unknown parameters and the most probable set of parameters by maximizing the log-likelihood function. This set describes the measured data well, as shown in Fig. 3b. In addition, the energy deposited in the fiber can be calculated. The combined fit of all proton data results in a value of $(0.127 \pm 0.030) \mathrm{mm} / \mathrm{MeV}$ for the Birks' coefficient of our scintillating fibers. We used this method to reconstruct the initial energies of particle beams with precision in the sub-MeV range for the energy range of $37 \mathrm{MeV}$ to $63 \mathrm{MeV}$. We take this as an upper limit on the energy resolution for protons of the MAPT detector.

\section{Summary}

The AFIS mission will measure the flux of trapped low-energy antiprotons in the inner Van Allen belt. To fulfill all the requirements for applications on nanosatellites, we developed an activetarget particle detector comprised of scintillating plastic fibers and silicon photomultipliers. The detector is sensitive to antiproton energies below $100 \mathrm{MeV}$ and has a large geometrical acceptance of about $250 \mathrm{~cm}^{2} \mathrm{sr}$. Hence, it will be possible to collect a statistically significant dataset to measure the flux of low-energy antiprotons in the SAA. Antiproton identification relies on a combination of Bragg-curve spectroscopy and the identification of the characteristics of antiproton annihilation.

A scaled-down prototype was built to investigate the response of the scintillating fibers and SiPMs to ionizing radiation and stopping protons. We tested it in a particle beam and concluded that it is possible to detect both minimum-ionizing radiation and highly ionizing radiation. Analysis of the data revealed two major processes affecting the reconstruction of energy deposited in the detector volume: ionization quenching of the plastic scintillators and saturation effects in the SiPMs. By comparing numerical simulations to experimental data, we quantified these effects and reconstructed the initial energies of the stopped protons. We determined the initial energies of the protons with an uncertainty of less than $1 \mathrm{MeV}$ in the energy range of $37 \mathrm{MeV}$ to $63 \mathrm{MeV}$. This value can be used as an estimate of the upper limit of the resolution of the detector for protons. The analysis method based on Bayesian inference techniques will be extended to single event analysis and to provide particle identification for the AFIS mission. 


\section{Acknowledgments}

This research was supported by the DFG Cluster of Excellence Origin and Structure of the Universe.

\section{References}

[1] Adriani, O., Barbarino, G. C., Bazilevskaya, G. a., et al. (2011). The Discovery of Geomagnetically Trapped Cosmic-Ray Antiprotons. The Astrophysical Journal, 737(2):L29.

[2] Barkas, W. H., Dyer, J. N., \& Heckmann, H. H. (1963). Resolution of the $\varepsilon^{-}$-Mass Anomaly. Physical Review Letters, 11(1):26-28.

[3] Berger, M. J., Coursey, J. S., \& Zucker, M. A. (1999). ESTAR, PSTAR, and ASTAR: Computer Programs for Calculating Stopping-Power and Range Tables for Electrons, Protons, and Helium Ions (version 1.21).

[4] Birks, J. B. (1964). The Theory and Practice of Scintillation Counting. Pergamon Press.

[5] Caldwell, A., Kollár, D., \& Kröninger, K. (2009). BAT âĂŞ The Bayesian analysis toolkit. Computer Physics Communications, 180(11):2197-2209.

[6] Fuki, M. (2005). Cosmic-Ray Antiproton Spatial Distributions Computed In Magnetosphere. International Journal of Modern Physics A, 20(29):6739-6741.

[7] Gruhn, C., Binimi, M., Legrain, R., et al. (1982). Bragg curve spectroscopy.

[8] Gusev, A., Pugacheva, G., Pankov, V., et al. (2008). Antiparticle content in the magnetosphere. Advances in Space Research, 42(9):1550-1555.

[9] Illingworth, J. \& Kittler, J. (1988). A Survey of the Hough Transform. Computer Vision, Graphics, and Image Processing, 44(1):87-116.

[10] Knoll, G. F. (1979). Radiation Detection and Measurement. Wiley.

[11] Losekamm, M. J., Pöschl, T., Langer, M., \& Paul, S. (2014). The AFIS Detector: Measuring Antimatter Fluxes on Nanosatellites. In 65th International Astronautical Congress.

[12] Mann, A. B., Konorov, I., Angerer, H., et al. (2009). The universal sampling ADC readout system of the COMPASS experiment. In IEEE Nuclear Science Symposium Conference Record, pages 2225-2228.

[13] Maus, S., Macmillan, S., McLean, S., et al. (2010). The US/UK World Magnetic Model for 2010-2015. NOAA Technical Report NESDIS/NGDC.

[14] Selesnick, R. S., Looper, M. D., Mewaldt, R. A., \& Labrador, A. W. (2007). Geomagnetically trapped antiprotons. Geophysical Research Letters, 34(20):2-5.

[15] Tadday, K. A. (2011). Scintillation Light Detection and Application of Silicon Phtomultipliers in Imaging Calorimetry and Positron Emission Tomography. $\mathrm{PhD}$ thesis, University of Heidelberg.

[16] Terasa, C., Labrenz, J., Kühl, P., et al. (2013). Extension of measurement capabilities of the Electron Proton Helium INstrument aboard SOHO. In 33nd International Cosmic Ray Conference, number 1 .

[17] The CubeSat Program (2014). CubeSat Design Specification. California Polytechnic State University, Rev. 13.

[18] Van Allen, J. A. (1958). Observation of High Intensity Radiation by Satellites 1958 Alpha and Gamma. Journal of Jet Propulsion, 28(9):588-592.

[19] Van Allen, J. A. \& Frank, L. A. (1959). Radiation Around the Earth to a Radial Distance of $107,400 \mathrm{~km}$. Nature, 183(4659):430-434. 